\title{
A SURVEY REGARDING PREFERENCE IN MANAGEMENT OF BILATERAL STONE DISEASE and COMPARISON OF CLAVIEN COMPLICATION RATE IN BILATERAL VERSUS UNILATERAL PERCUTANEOUS NEPHROLITHOTOMY
}

Marcelino E. Rivera ${ }^{1}$, Naeem Bhojani ${ }^{2}$, Kevin Heinsimer ${ }^{1}$, Marawan M. EI Tayeb $^{3}$, Jessica E. Paonessa ${ }^{4}$, Amy E. Krambeck ${ }^{1}$ and James E. Lingeman ${ }^{1}$

${ }^{1}$ Indiana University School of Medicine, Indianapolis, IN, USA

2 University of Montreal, Montreal, QC, Canada

3 Baylor Scott and White Health

4 SUNY Upstate Medical Center

\begin{abstract}
Address all correspondence to:
James E. Lingeman MD

Indiana University School of Medicine

1801 Senate Blvd. Suite 220

Indianapolis, IN

46202

Tel: 317.962 .2485

Fax: 317.962.2893

Email: JLingeman@iuhealth.org

Word Count: 2892

Abstract: 250

Text: 2642

Tables: 3

Figures: 1

Running head: Bilateral percutaneous nephrolithotomy complications

Key Words: Percutaneous, nephrolithotomy, bilateral, Clavien, complications
\end{abstract}

This is the author's manuscript of the article published in final edited form as:

Rivera, M. E., Bhojani, N., Heinsimer, K., El Tayeb, M. M., Paonessa, J. E., Krambeck, A. E., \& Lingeman, J. E. (2017). A Survey Regarding Preference in Management of Bilateral Stone Disease and Comparison of Clavien Complication Rate in Bilateral Versus Unilateral Percutaneous Nephrolithotomy. Urology. https://doi.org/10.1016/j.urology.2017.09.020 
Abstract

Purpose: To discuss complications of simultaneous bilateral percutaneous nephrolithotomy (SB-PCNL) when compared to unilateral percutaneous nephrolithotomy (U-PCNL) and survey surgeon preference in bilateral stone disease management.

Materials and Methods:

A database of all participating PCNL patients who received treatment at Indiana University Health Methodist Hospital within a 10-year period from 2006 to 2015 by a single surgeon (JL) was utilized. Perioperative data as well as complications, as defined according the Clavien grading system, were recorded. A survey of members of the Endourological Society was performed regarding surgical management in the setting of bilateral stone disease.

Results

A total of 563 patients were identified over the study period with 129 undergoing SB-PCNL. Overall, SB-PCNL patients had a longer procedure (176.9 vs115.6 $\min , p<0.0001)$, were more likely to undergo a secondary procedure $(73 \%$ vs 44 $p<0.001$ ), and had a longer hospital stay ( 3.2 vs 2.3 days, $p<0.001)$. Notably, there were no differences in number or severity of complications between the two groups.

A total of 153 endourologists completed the survey. Of these $58(38 \%)$ perform bilateral PCNL under anesthesia. The top reasons for electing to not perform 
bilateral PCNLs included: duration of bilateral procedures (53\%), bilateral renal injury (48\%), rarely perform bilateral surgery $(35 \%)$.

\section{Conclusions}

Although procedure length was longer in the SB-PCNL group there were similar rates of complications and severity between U-PCNL and SB-PCNL. A majority of endourologists surveyed do not perform bilateral PCNL but would perform bilateral URS with duration of the procedure and concern for bilateral renal injury representing the most common reasons.

Introduction

Since the first PCNL in 1976, percutaneous nephrolithotomy (PCNL) has become the standard of care for treatment of large and complex nephrolithiasis. ${ }^{1}$ While recent reports have demonstrated that PCNL is a safe procedure with acceptable post-operative complication rates, there are certain circumstances where there is a theoretical increased risk of complications. ${ }^{2} 34$ Bilateral stone disease presents a unique challenge to the treating urologist, in particular when there is significant stone burden warranting PCNL. SB-PCNL was first reported by Colon-Perez in 1987 and while there is a hypothetical increased risk to simultaneous bilateral PCNL (SB-PCNL), multiple investigations have demonstrated its safety and efficacy as well as decreased cost in the complex stone patient ${ }^{5-9}$. However, a study from Hungary did report an increased risk of complications with SB-PCNL compared to unilateral PCNL (U-PCNL). ${ }^{10}$ 
Although complication rates associated with PCNL in the reported literature are low, classification of these complications can be difficult as there are currently many surgical technique variations, including radiologist assisted access as well as other ancillary procedures, utilization of lithotripsy and scope technology, and surgical approaches, which can lead to varied interpretation of what constitutes a complication. ${ }^{2}$ With this in mind, recent publications have aimed to categorize complications utilizing the widely accepted modified Clavien classification. ${ }^{11}$ Although there have been reports utilizing the modified complication classification system comparing SB-PCNL and U-PCNL, these have typically been small series. The lack of robust literature regarding this clinical question led to the development of a survey submitted to the Endourological Society email listserv. Therefore, we aim to add to the current body of literature with a large institutional series and to discuss surgeon preference and rationale regarding bilateral stone disease cases.

Patients and methods Patients

A prospectively maintained database of all participating PCNL patients who received treatment at Indiana University Health Methodist Hospital within a 10-year period from 2006 to 2015 by a single surgeon (JEL) was utilized for analysis. Preoperative data including age, gender, comorbidities, anatomical anomalies, and preoperative serum creatinine and hemoglobin levels were recorded. Additionally, intraoperative data including stone size, total surgical 
time, length of stay, and secondary procedures were documented. Finally, postoperative data, including serum creatinine and hemoglobin levels at 24 hours after surgery, and complications, as defined according the Clavien grading system, were recorded.

\section{Technique}

Following the placement of a $5 \mathrm{Fr}$ ureteral and bladder catheters, the patient was placed in the prone position. Renal access was obtained using an 18-gauge diamond tip access needle with the number and location of access points decided based on the anatomy of the kidney as well as the complexity of the stones. Renal access was obtained by the urologist using a triangulation technique. After verification of access with urine aspiration, a 0.035-inch hydrophilic guidewire was passed through the needle and into the renal collecting system. In most situations the guidewire, after being positioned in the ureter, was exchanged for an Amplatz super stiff working wire; a second safety wire was also placed down the ureter. Finally, balloon dilatation was performed and a $30 \mathrm{Fr}$ working sheath was placed into the calyx of interest. ${ }^{12}$ For bilateral PCNL, a $5 \mathrm{Fr}$ ureteral catheter was placed in each of the two ureters at the beginning of the procedure. The more complex side was always treated first.

A combination of rigid and flexible nephroscopy was performed, and lithotripsy was carried out using a combination of ultrasonic, pneumatic, and holmium laser energy. In most cases of PCNL, a 10 Fr Cope loop catheter was left in the renal collecting system and a $5 \mathrm{Fr}$ open-ended catheter was placed 
antegradely in the ureter. All patients had a blood draw on postoperative day (POD) 1 as well as a noncontrast CT scan. All patients with fragments on their CT scan underwent a secondary procedure on POD 2. Nephrostomy tube removal was attempted on postoperative day one if the CT indicated that the renal unit was stone free. If residual fragments remained after a secondary procedure, patients underwent a third procedure to render them stone free.

Complications within 30 days of the procedure were recorded and classified according to the Clavien system of grading complications with the categorization recommended by de la Rosette et al. ${ }^{11}$. All analyses were performed using JMP ${ }^{\circledR}$, Version 12.0. (SAS Institute Inc., Cary, NC, 1989-2007), using two-tailed testing with a significance level of 0.05 .

Results

\section{Preoperative characteristics}

A total of 563 patients were identified over the study period with 129 undergoing SB-PCNL. Clinical baseline characteristics are presented in Table 1. Mean age was 53 (IQR 43-65) and 54 (IQR 44-65) years, $p=0.60$ with mean BMI of 32.0 (IQR 25.1-36.6) and 32.1 (IQR 21.3-39), p=0.89 between the U-PCNL and SBPCNL groups, respectively. There was a significant difference in baseline renal function between the two groups with mean serum creatinine values of 1.02 (IQR, 0.79-1.13) and 1.13 (IQR, 0.84-1.25), p=0.02 for U-PCNL and SB-PCNL, respectively. Likewise, the diagnosis of gout $(p=0.01)$ and horseshoe kidney $(p=0.04)$ was significantly higher in the SB-PCNL cohort. Intra- and postoperative characteristics 
Overall, patients who underwent SB-PCNL had a longer duration of their procedure $(176.9$ vs115.6, $p<0.001)$, were more likely to undergo a secondary procedure $(73 \%$ vs $44, p<0.001)$, even when stratified on a renal unit basis $(62 \%$ vs $44, \mathrm{P}<0.001)$. Thus, the stone free rate after the primary procedure was 27 vs $56 \%(p<0.001)$ in the SB-PCNL and U-PCNL groups, respectively. The SBPCNL group had a longer length of hospitalization (3.2 vs 2.3 days, $p<0.001)$. The procedure was terminated early once in each group, due to return of purulence with initial puncture in the SB-PCNL and hemorrhage in U-PCNL group. We also identified a conversion from contralateral URS with U-PCNL to SB-PCNL in three cases. Similarly, renal function at 24 hours post-op (1.43 vs 1.23, $p=0.002)$ and mean hemoglobin (11.2 vs11.7 $p=0.007)$ were both significantly decreased in the SB-PCNL group compared to the U-PCNL cohort. By $48 \mathrm{hrs}$ post-operatively, the renal function was no longer significantly different between the two groups $(p=0.15)$. The proportion of infectious stones was similar between the two groups with $10 \%$ of both cohorts having infectious stones, struvite or carbonate apatite, on analysis. The numbers of complications between the two groups were not statistically different with $33 \%$ of the SB-PCNL cohort and $27 \% \quad(p=0.15)$ of the U-PCNL experiencing a complication (Table 2). Likewise, within the complication group there were no differences in severity of complications when comparing the $U$ and SB-PCNL groups.

\section{Survey Results}


Regarding the survey to the Endourological Society, a total of 153 urologists completed the survey. Of these, 58 (38\%) perform bilateral PCNL under the same anesthesia, 74 (48\%) perform bilateral URS but not bilateral PCNL, and 21 (14\%) do not perform bilateral stone procedures under the same anesthetic. Those who do not perform bilateral PCNL were willing to offer their patients staged PCNLs (88\%), unilateral PCNL and contralateral URS (10\%) and/or unilateral PCNL with observation of the contralateral side (5\%). Reasons for electing to not perform bilateral PCNLs included: rarely perform bilateral renal stone surgery $(35 \%)$, duration of bilateral procedures $(53 \%)$, bilateral renal injury (48\%), bleeding risk (34\%), patient discomfort (23\%), reimbursement (13\%), burden of bilateral procedure to operating room staff (11\%), and hospital policy (6\%) (Figure 1). Urologists who were willing to do unilateral PCNL and contralateral URS $(n=9)$ felt their method of treatment was safer $(56 \%)$, had less risk of acute kidney injury (78\%), and less blood loss (11\%). Regarding bilateral ureteroscopy, 131 urologists (85\%) were willing to perform bilateral URS under the same anesthesia, while $14 \%$ would do staged URS, and $1 \%$ would do unilateral URS and observe the contralateral side.

Finally a clinical scenario of a patient with bilateral $2.5 \mathrm{~cm}$ stones, amenable to PCNL was given. When offered management options, those who perform bilateral PCNL, chose bilateral PCNL most often (74\%), staged PCNL (12\%), staged URS (5\%), bilateral URS (5\%), and unilateral PCNL with contralateral URS (4\%). Urologists who do not perform bilateral PCNLs elected to perform 
staged PCNL (83\%), staged URS (12\%), unilateral PCNL and contralateral URS (3\%), and bilateral URS (2\%).

\section{Discussion}

Over a ten-year period within a single surgeon experience of $U$ and SB-PCNL we identified no significant difference in the number of complications or severity of complications when using the CROES PCNL study group assigned Clavien complication grading system. The vast majority of our complications were grade I or II. While patients undergoing SB-PCNL did have lower postoperative hemoglobins on the first postoperative day, this was not clinically significant as there was no difference in transfusion rates between groups. Furthermore the SB-PCNL group had a higher baseline creatinine compared to the U-PCNL so the difference in renal function between groups is minimal. As expected, the procedure length and hospital stay was significantly longer and rate of secondary procedures was likewise higher in the SB-PCNL cohort. However, procedure length was not twice that of U-PCNL and thus a more efficient procedure for the patient. There was a single Clavien $\mathrm{V}$ complication in a patient who was found to have widely metastatic colorectal cancer shortly after his procedure and was placed on hospice and died within 30 days of his procedure. 
We also found that the majority of endourologists surveyed do not offer bilateral PCNL to their patients as an option for treatment. The most common concerns for not performing bilateral procedures were the duration of the procedure, concern for bilateral renal injury and rarely performing the procedure. While a recent survey investigation of endourologists queried other patient management topics, ${ }^{13}$ to our knowledge this is the first survey to the Endourological field investigating procedural preference in the setting of bilateral stone disease.

A recent systematic review by Jones et al into the safety and feasibility of SBPCNL notes only 11 investigations with a total of 594 patients meeting inclusion criteria over a span of 18 years. ${ }^{14}$ While our patients were significantly older than the review, rates of complications were similar. Interestingly, the most common complication in this review was fever but the complications were reported utilizing the modified Clavien-Dindo classification and likely underestimating the true number of complications. Our investigation would add significantly to the current body of literature in both procedure number and by better defining procedure specific complications.

Clavien was the first to propose a reporting system to define and classify surgical complications to allow for standardized reporting throughout the literature. ${ }^{15}$ In 2004, Dindo et al. evaluated the reproducibility of the modified Clavien system and found that it correlated well with complexity of surgery and hospital stay. The Clavien system serves as the guideline for classification of postoperative 
morbidity and mortality to this day. ${ }^{16}$ The creation of a surgical complications reporting system has led to the more objective reporting of patient outcomes in the literature. However, the Clavien system is not specialty specific and thus can lead to inconsistent reporting of the severity of postoperative complications. The limitations in reporting of complications in PCNL was noted in a recent review by Seitz et al. and the authors recommended a modified Clavien system be established. ${ }^{2}$ Therefore, de la Rossette and the Clinical Research Office of the Endourology Society (CROES) analyzed the interrater reliability of PCNL complications. After surveying a group of urologists who rated a set of complications the authors found low reliability between individuals with regards to lower Clavien complications. In order to reduce this variability in the future, a proposed categorization of complications based on the Clavien system was constructed based on expert opinion. ${ }^{11}$ This CROES procedure-specific grading system was utilized to define all complications within our investigation.

SB-PCNL was first reported nearly 10 years after initial U-PCNL reports; however, ${ }^{51718}$ there is limited data comparing the complications associated between SB-PCNL and U-PCNL. Looking only at U-PNCL, the CROES PCNL Global Study database with 5724 patients reported a complication rate of $20.5 \%$ using the Clavien complication grading system. ${ }^{19}$ Desai et al in 2007 demonstrated a complication rate of $22.2 \%$ in 45 patients undergoing SBPCNL. ${ }^{20}$ Holman et al in 2002 compared 150 SB-PCNLs with 300 U-PCNLs. $^{9}$ This study found complications rates of $14.3 \%$ and $11.3 \%$ for patients undergoing 
SB-PCNL and UPCNL, respectively. Silverstein et al in 2004 compared 19 patients who underwent staged U-PCNLs to 17 patients who underwent SBPCNLs. ${ }^{6}$ They reported complication rates of $19 \%$ and $28 \%$ for the staged and simultaneous PCNL groups, respectively. They noted less blood loss in patients undergoing SB-PCNL compared to those in a staged approach. However, transfusion rates while comparable were noted to be considerable between the two groups at 28.6 and $36.8 \%$ (p-value=NS) for the SB-PCNL and the asynchronous group, respectively. The transfusion rate of our cohort was not significantly different at $2 \%$ and $4.7 \%$ for U-PCNL and SB-PCNL respectively. While overall complication rates were not discussed, blood loss may serve as an indicator of the similar morbidity that SB-PCNL has to U-PCNL.

Although multiple reports have indicated similar morbidity between SB-PCNL and U-PCNL, investigators have raised caution in certain circumstances such as complex renal anatomy. ${ }^{19}$ Our investigation did find a difference in complex renal anatomy with a significantly higher number of patients undergoing SB-PCNL who had a horseshoe kidney. Nonetheless, there were no statistically significant differences within the cohort even with the added anatomic complexity. A recent investigation would seem to disagree with the notion of SB-PCNL and U-PCNL having similar rates of complications. Kadlec et al., in an institutional series of 47 patients over 11 years who underwent bilateral PCNL, noted an increase in any complication within the bilateral PCNL group when compared to a matched unilateral cohort, with over $50 \%$ having a complication compared to $31 \%(p=0.01)$ in the U-PCNL group. ${ }^{10}$ Renal access was obtained by a 
radiologist the day prior to the planned procedure. While the analysis of Kadlec et al. utilized the Clavien classification, it did not apply the PCNL specific categorization recommended by the CROES group and did not discuss prolonged nephrostomy drainage, the most common complication in our cohort. While the current investigation is over a similar period of time, we report a considerably larger cohort of patients. Also dissimilar to our series was a rather high rate of sepsis in the bilateral cohort (6.4\%) of the Kadlec et al study. In our series we noted a sepsis rate of approximately $1 \%$ in the entire cohort which may in turn be due to our perioperative antibiotic protocol. This includes a minimum of 7 days pre- and 7 days of post-procedural antibiotics. Overall, we note fewer complications with no difference in the severity of complications within our series.

While our study represents the largest single series in the literature, it is not without limitations. Our survey results are prone to response bias and may not represent the entirety of the Endourological society. Although our rates of complications are similar to that of the reported literature, we are the first to utilize the CROES categorization of complications and thus, may have a higher rate of Clavien grade 1 and 2 complications. Our medical center serves as a high volume tertiary referral center for stone disease and therefore, the generalizability of our patients and outcomes may not reflect that of a smaller referral practice. Finally, although the series represents that of an experienced surgeon, it should be noted that as a tertiary teaching hospital residents and fellows participated in all aspects of the cases including percutaneous access. 
Conclusion

Although U-PCNL and SB-PCNL did have significant differences in certain reported metrics including total operative time, likelihood for a secondary procedure, and length of hospital stay, rates of complications and severity of complications were similar between the two groups. Thus, SB-PCNL should be considered an acceptable treatment alternative for patients with bilateral stone burden warranting percutaneous management. We also identified that a majority of endourologists surveyed do not perform bilateral PCNL but would perform bilateral URS, citing the duration of the procedure and concern for bilateral renal injury representing the most common reasons.

\section{References}

1. Fernstrom I, Johansson B. Percutaneous pyelolithotomy. A new extraction technique. Scand J Urol Nephrol 1976;10(3):257-9.

2. Seitz C, Desai M, Hacker A, et al. Incidence, prevention, and management of complications following percutaneous nephrolitholapaxy. Eur Urol 2012;61(1):146-58. doi: 10.1016/j.eururo.2011.09.016

3. de la Rosette J, Assimos D, Desai M, et al. The Clinical Research Office of the Endourological Society Percutaneous Nephrolithotomy Global Study: indications, complications, and outcomes in 5803 patients. J Endourol 2011;25(1):11-7. doi: 10.1089/end.2010.0424

4. Labate G, Modi P, Timoney A, et al. The percutaneous nephrolithotomy global study: classification of complications. J Endourol 2011;25(8):1275-80. doi: 10.1089/end.2011.0067

5. COLÓN-PÉREZ BC, R. J.; and RAMOS, M. E. Simultaneous Bilateral Nephrostolithotomies: Immediate Results in Three Cases. Journal of Endourology 1987;1(3):209-13. doi: 10.1089/end.1987.1.209 [published Online First: March 2009, 1(3): 209-213]

6. Silverstein AD, Terranova SA, Auge BK, et al. Bilateral renal calculi: assessment of staged v synchronous percutaneous nephrolithotomy.J Endourol 2004;18(2):145-51. doi: 10.1089/089277904322959770 
7. Williams SK, Hoenig DM. Synchronous bilateral percutaneous nephrostolithotomy. J Endourol 2009;23(10):1707-12. doi: 10.1089/end.2009.1538

8. Bagrodia A, Raman JD, Bensalah K, et al. Synchronous bilateral percutaneous nephrostolithotomy: analysis of clinical outcomes, cost and surgeon reimbursement. J Urol 2009;181(1):149-53. doi: 10.1016/j.juro.2008.09.011

9. Holman E, Salah MA, Toth C. Comparison of 150 simultaneous bilateral and 300 unilateral percutaneous nephrolithotomies. J Endourol 2002;16(1):33-6. doi: 10.1089/089277902753483691

10. Kadlec AO, Greco KA, Fridirici ZC, et al. Comparison of complication rates for unilateral and bilateral percutaneous nephrolithotomy (PCNL) using a modified Clavien grading system. BJU Int 2013;111(4 Pt B):E243-8. doi: 10.1111/j.1464-410X.2012.11589.x

11. de la Rosette JJ, Opondo D, Daels FP, et al. Categorisation of complications and validation of the Clavien score for percutaneous nephrolithotomy. Eur Urol 2012;62(2):246-55. doi: 10.1016/j.eururo.2012.03.055

12. Miller NL, Matlaga BR, Lingeman JE. Techniques for fluoroscopic percutaneous renal access. J Urol 2007;178(1):15-23. doi: 10.1016/j.juro.2007.03.014 [published Online First: 2007/06/19]

13. Becker B, Knipper S, Gross AJ, et al. Current Management in Transurethral Therapy of Benign Prostatic Obstruction in Patients on Oral Anticoagulation: A Worldwide Questionnaire. J Endourol 2017;31(2):163-68. doi: 10.1089/end.2016.0627

14. Jones P, Dhliwayo B, Rai BP, et al. Safety, Feasibility, and Efficacy of Bilateral Synchronous Percutaneous Nephrolithotomy for Bilateral Stone Disease: Evidence from a Systematic Review. J Endourol 2017;31(4):334-40. doi: 10.1089/end.2016.0851

15. Clavien PA, Sanabria JR, Strasberg SM. Proposed classification of complications of surgery with examples of utility in cholecystectomy. Surgery 1992;111(5):518-26.

16. Dindo D, Demartines N, Clavien PA. Classification of surgical complications: a new proposal with evaluation in a cohort of 6336 patients and results of a survey. Ann Surg 2004;240(2):205-13.

17. Ahlawat R, Banerjee GK, Dalela D. Bilateral simultaneous percutaneous nephrolithotomy. A prospective feasibility study. Eur Urol 1995;28(2):116-8.

18. Dushinski JW, Lingeman JE. Simultaneous bilateral percutaneous nephrolithotomy. J Urol 1997;158(6):2065-8.

19. Labate G, Modi P, Timoney A, et al. The percutaneous nephrolithotomy global study: classification of complications. J Endourol 2011;25(8):1275-80. doi: 10.1089/end.2011.0067 [published Online First: 2011/07/15]

20. Desai M, Grover R, Manohar T, et al. Simultaneous bilateral percutaneous nephrolithotomy: a single-center experience. J Endourol 2007;21(5):508-14. doi: 10.1089/end.2006.0401 [published Online First: 2007/05/26] 
Figure 1.| Graph of Survey results regarding respondents who do not offer B/L PCNL

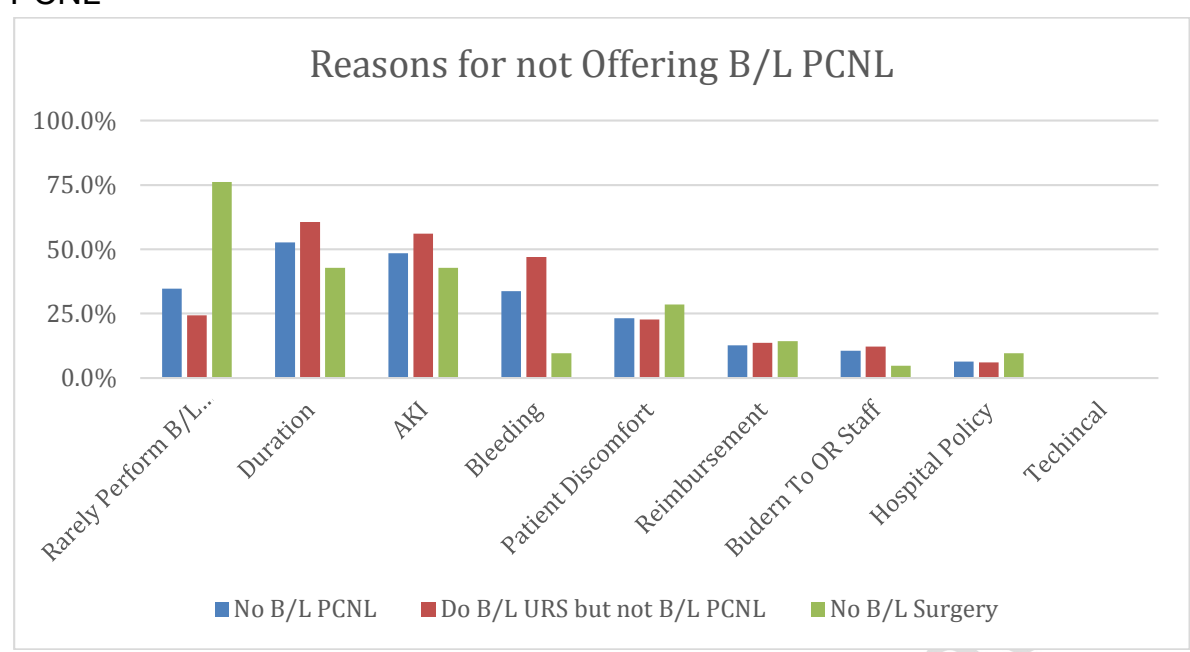


Table 1. Preoperative demographics of patients who underwent unilateral or simultaneous bilateral percutaneous nephrolithotomy $\mathrm{n}=$ number of patients

\begin{tabular}{|c|c|c|c|}
\hline & $\begin{array}{c}\text { Unilateral } \\
(\mathrm{n}=434)\end{array}$ & Bilateral (n=129) & $P$ value \\
\hline $\begin{array}{c}\text { Years of age (mean } \\
\pm \text { SD) }\end{array}$ & $53.4(15.7)$ & $54.3(15.3)$ & 0.60 \\
\hline Male gender (\%) & 45 & 49 & 0.42 \\
\hline BMI (mean \pm SD) & 32.0 & 32.1 & 0.89 \\
\hline $\begin{array}{c}\text { Pre-operative serum } \\
\text { Creatinine } \\
\text { (mg/dl, mean } \pm \text { SD) }\end{array}$ & $1.02(0.4)$ & $1.13(0.5)$ & 0.02 \\
\hline $\begin{array}{c}\text { Pre-operative Hb } \\
\text { (g/dl, mean } \pm \text { SD) }\end{array}$ & $13.7(1.69)$ & $13.8(1.79)$ & 0.69 \\
\hline UTI (\%) & $48 \%$ & $52 \%$ & 0.37 \\
\hline Hypertension (\%) & $47 \%$ & $45 \%$ & 0.61 \\
\hline Diabetes (\%) & $23 \%$ & $24 \%$ & 0.81 \\
\hline $\begin{array}{c}\text { Gout (\%) } \\
\text { Hyperparathyroidism } \\
(\%)\end{array}$ & $3 \%$ & $9 \%$ & 0.01 \\
\hline $\begin{array}{c}\text { Horseshoe Kidney } \\
(\%)\end{array}$ & $2 \%$ & $4 \%$ & 0.04 \\
\hline $\begin{array}{c}\text { Staghorn Calculus } \\
(\%)\end{array}$ & $45 \%$ & $6 \%$ & 0.31 \\
\hline $\begin{array}{c}\text { Calyceal } \\
\text { diverticulum (\%) }\end{array}$ & $5 \%$ & $40 \%$ & 0.09 \\
\hline $\begin{array}{c}\text { Left Stone Burden } \\
>2 \text { 2cm (\%) }\end{array}$ & $82 \%$ & $2 \%$ & 0.50 \\
\hline $\begin{array}{c}\text { Right Stone Burden } \\
>2 \text { 2cm (\%) }\end{array}$ & $77 \%$ & $79 \%$ & 0.11 \\
\hline $\begin{array}{c}\text { Urinary Diversion } \\
(\%)\end{array}$ & $2 \%$ & $70 \%$ & 0.0 \\
\hline Paresis (\%) & $3 \%$ & $2 \%$ & 0.04 \\
\hline
\end{tabular}


Table 2. Postoperative characteristics and complications of patients who underwent unilateral or bilateral percutaneous nephrolithotomy

\begin{tabular}{|c|c|c|c|}
\hline & $\begin{array}{l}\text { Unilateral } \\
(n=434)\end{array}$ & Bilateral $(n=129)$ & $P$ value \\
\hline $\begin{array}{l}\text { Creatinine, } 24 \mathrm{hrs} \text { post-operative } \\
\text { (mg/dl, mean } \pm \mathrm{SD} \text { ) }\end{array}$ & $1.23(0.67)$ & $1.43(0.62)$ & 0.002 \\
\hline $\begin{array}{l}\text { Creatinine, } 48 \mathrm{hrs} \text { post-operative } \\
(\mathrm{mg} / \mathrm{dl} \text {, mean } \pm \text { SD) }\end{array}$ & $1.37(0.89)$ & $1.62(0.90)$ & 0.15 \\
\hline $\mathrm{Hb} @ 24 \mathrm{hrs}$ (g/dl, mean \pm SD) & $11.7(1.74)$ & $11.2(1.76)$ & 0.007 \\
\hline Transfusion (\%) & $2.0 \%$ & $4.7 \%$ & 0.11 \\
\hline $\begin{array}{l}\text { Length of stay (days; mean, } \\
\text { range) }\end{array}$ & $2.3(1-23)$ & $3.2(1-21)$ & $<0.001$ \\
\hline Total surgery time $(\mathrm{min})(\mathrm{SD})$ & $115.6(42.9)$ & $176.9(54.1)$ & $<0.0001$ \\
\hline Secondary procedures (\%) & 44 & 73 & $<0.0001$ \\
\hline $\begin{array}{l}\text { Renal units undergoing } \\
\text { secondary }(\%)\end{array}$ & 44 & 62 & $<0.0001$ \\
\hline \multicolumn{4}{|l|}{ Complications } \\
\hline Clavien \% (n) (overall) & $27(116)$ & $33(43)$ & 0.15 \\
\hline Clavien I & $17.8(77)$ & $19.4(25)$ & 0.36 \\
\hline Clavien II & $3.5(15)$ & $7.8(10)$ & 0.14 \\
\hline Clavien Illa & $0.9(4)$ & $3.1(4)$ & 0.83 \\
\hline Clavien IIIb & $2.5(11)$ & $0.8(1)$ & \\
\hline Clavien IVa & $1.2(5)$ & $0.8(1)$ & \\
\hline Clavien IVb & $0.7(3)$ & $1.6(2)$ & \\
\hline Clavien V & $0.2(1)$ & 0 & \\
\hline
\end{tabular}

Most frequent Clavien I complication: Delayed removal of nephrostomy tube Most frequent Clavien II complication: Blood loss requiring blood transfusion Most frequent Clavien III complication: Edema/clot retention requiring stent placement

Most frequent Clavien IV complication: Sepsis 
Table 3. Detailed list of complications by Clavien classification

\begin{tabular}{|c|c|}
\hline $\begin{array}{l}\text { Clavien } \\
\text { grade }\end{array}$ & Complications (U-PCNL/SB-PCNL) \\
\hline Grade I $\left(n^{\star}\right)$ & $\begin{array}{l}\text { Delayed removal or displacement of nephrostomy tube (32/9) } \\
\text { Blood loss anemia without need for transfusion (10/2) } \\
\text { Elevated postoperative temperature }\left(>38^{\circ} \mathrm{C}\right) \text { managed without } \\
\text { antibiotics }(8 / 7) \\
\text { Subcapsular hematoma and/or perinephric hematoma }(6 / 0) \\
\text { Small collecting system perforation }(6 / 0) \\
\text { Post-operative pain managed with adjunct opioid analgesics }(5 / 4) \\
\text { Urinary retention (4/0) } \\
\text { Hypotension (3/1) } \\
\text { Electrolyte derangement managed conservatively (1/0) } \\
\text { Hydrothorax managed expectantly (1/0) } \\
\text { Desaturation responding to conservative management }(1 / 0) \\
\text { Pneumothorax managed with watchful waiting (0/1) }\end{array}$ \\
\hline Grade II $\left(n^{*}\right)$ & $\begin{array}{l}\text { Bleeding requiring transfusions or receipt of blood product }(5 / 6) \\
\text { Fever managed with antibiotics }(4 / 1) \\
\text { Deep vein thrombosis }(3 / 0) \\
\text { Cardiac arrhythmia }(2 / 0) \\
\text { Hyposaturation managed by oxygen therapy }(1 / 1) \\
\text { lleus with conservative management }(0 / 1)\end{array}$ \\
\hline $\begin{array}{l}\text { Grade IIla } \\
\left(n^{*}\right)\end{array}$ & $\begin{array}{l}\text { Embolization for pseudoaneurysm and arteriovenous fistula (2/1) } \\
\text { Hydro/pneumothorax managed by drain/tube placement (1/1) } \\
\text { Renal abscess treated with aspiration (0/1) } \\
\text { UTI without organ failure requiring advanced monitoring (1/1) }\end{array}$ \\
\hline
\end{tabular}




\begin{tabular}{|l|l|}
\hline $\begin{array}{l}\text { Grade IIIb } \\
\left(n^{*}\right)\end{array}$ & $\begin{array}{l}\text { Stent placement for edema/clot }(6 / 1) \\
\text { Bladder clot evacuation }(3 / 0) \\
\text { Colonic injury }(2 / 0)\end{array}$ \\
\hline $\begin{array}{l}\text { Grade IVa } \\
\left(n^{*}\right)\end{array}$ & $\begin{array}{l}\text { Respiratory failure (3/1) } \\
\text { Acute Renal Failure requiring ICU management }(2 / 0)\end{array}$ \\
\hline $\begin{array}{l}\text { Grade IVb } \\
\left(n^{*}\right)\end{array}$ & Sepsis and multi-organ failure $(3 / 2)$ \\
\hline Grade V & Death $(1 / 0)$ \\
\hline
\end{tabular}

